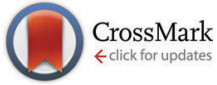

Cite this: J. Mater. Chem. B, 2015, 3, 6520

\section{Liposome-induced exfoliation of graphite to few-layer graphene dispersion with antibacterial activity $\dagger$}

\author{
R. Zappacosta, ${ }^{a}$ M. Di Giulio, ${ }^{a}$ V. Ettorre, ${ }^{a}$ D. Bosco, ${ }^{\text {b }}$ C. Hadad, ${ }^{C}$ G. Siani, ${ }^{a}$ \\ S. Di Bartolomeo, ${ }^{a}$ A. Cataldi, ${ }^{a}$ L. Cellini*a and A. Fontana*a
}

\begin{abstract}
Few-layer graphene aqueous dispersions are obtained by exploiting liposomes as effective exfoliating agents for graphite. Raman measurements evidence the presence of non-oxidized double layer graphene as well as amphiphilic phospholipid molecules organized in bilayers in the samples. TEM analyses confirmed that the obtained homogeneous graphene nanosheets are embedded in the liposomal bilayer. The as-prepared graphene aqueous dispersion is stable for days and demonstrates significant antibacterial activity against both Gram-positive (Staphylococcus aureus) and Gram-negative (Escherichia coli) strains, with a reduction in the growth of S. aureus and E. coli as high as 60 and $78 \%$, respectively.
\end{abstract}

Received 30th April 2015,
Accepted 7th July 2015

DOI: $10.1039 / c 5 t b 00798 d$

www.rsc.org/MaterialsB

\section{Introduction}

In recent years a growing interest within the scientific community has been devoted to the study of carbon allotropes and their applications. Among them, graphene and its derivatives, due to their unique physicochemical properties, have aroused interest ${ }^{1-3}$ in many research fields. As a matter of fact, applications in electronic ${ }^{4-7}$ and photonic devices, ${ }^{8-10}$ clean energy, ${ }^{1,11,12}$ energy storage ${ }^{13}$ and sensors ${ }^{14,15}$ have been well demonstrated. In addition, graphene-based materials appear as promising scaffolds in biomedicine. ${ }^{1-3,16-18}$

For application in biomedicine, one of the most important and fundamental goal to be achieved is to make graphene soluble in water. Up to now, most of the research on graphene in the biomedical field has been focused on the production and characterization of hydrophilic graphene oxide (GO), i.e. graphene functionalized with hydroxyl, epoxy and carboxyl groups that render graphene suitable to interface with biological systems. As a matter of fact, the functionalization renders GO-based materials chemically versatile templates with high surfaceto-volume ratios and favours the realization of GO-based drug

\footnotetext{
a Dipartimento di Farmacia, Università 'G. d'Annunzio', Via dei Vestini, 66100 Chieti, Italy.E-mail: l.cellini@unich.it, fontana@unich.it

${ }^{b}$ Istituto di Genetica Molecolare, CNR unità di Chieti, I-66100 Chieti, Italy

${ }^{c}$ Center of Excellence for Nanostructured Materials (CENMAT), INSTM,

Unit of Trieste, Dipartimento di Scienze Chimiche e Farmaceutiche,

Università di Trieste, Via L Giorgieri 1, I-34127 Trieste, Italy

$\dagger$ Electronic supplementary information (ESI) available: Stability measurements; Raman fitting; precipitation of graphene embedded in liposomes; and Gram staining images. See DOI: 10.1039/c5tb00798d
}

delivery vehicles, ${ }^{19-21}$ biosensors, ${ }^{22-26}$ imaging agents ${ }^{19,27}$ and electromechanical devices for monitoring cellular responses. ${ }^{28}$

Besides GO demonstrates good biocompatibility with animal cells, ${ }^{29}$ while stimulating human mesenchymal stem cells to differentiate into osteoblasts, ${ }^{30-32}$ adipocytes $^{30}$ and myoblasts, $, 33,34$ inducing the differentiation of preosteoblasts into osteoblasts, ${ }^{35,36}$ neuronal stem cells into neurons ${ }^{37}$ and pluripotent stem cells into endodermal lineage. ${ }^{38}$ For this reason GO-based materials have been studied for tissue- and osteo-regeneration. ${ }^{39-41} \mathrm{GO}$ demonstrated to exhibit strong antibacterial activity towards both Gram-positive and Gram-negative bacteria. ${ }^{42,43}$

Functionalization of graphene to obtain GO has revealed undisputed advantages, but it involves the breakdown of the continuous honeycomb backbone of pristine (non-functionalized) graphene compromising several of the peculiar properties of the original material.

In order to preserve the structural integrity of graphene and use an absolutely green process of exfoliation and functionalization, inspired by Samori et $a l .{ }^{44}$ who demonstrated the excellent ability of fatty acids to exfoliate graphite in organic solvents and Titov's molecular dynamics simulation ${ }^{45}$ who showed the theoretical insertion of a "graphene sheet in the hydrophobic interior of biological membranes", in the present study, we proceeded to obtain nanosheets of graphene sandwiched between phospholipid alkyl chains by simply sonicating graphite in liposomal suspensions. The as-prepared aqueous dispersions demonstrate to solubilize as much as $161 \mu \mathrm{g} \mathrm{mL} \mathrm{m}^{-1}$ few-layer graphene in line with dispersions obtained with the use of classical surfactants such as sodium cholate but three times more concentrated than dispersions obtained with sodium dodecylbenzene sulfonate. ${ }^{46}$ The advantage of the present dispersions is that the flakes 
are relatively small i.e. (in the nanometric range) and ready to be used for different biomedical applications.

Considering that (i) resistance to antibiotics represents the major cause of treatment failure in bacterial infections, (ii) alternative proposals are needed due to overuse or misuse of common antimicrobials that compromises the therapeutic effect of traditional treatments ${ }^{47}$ and (iii) the proved antibacterial activity of the covalently functionalized oxidized analogue GO, we propose the as-prepared liposomal graphene-loaded aqueous dispersion as an innovative efficacious antibacterial system to overcome the alarming bacterial resistance increase. ${ }^{48,49}$ Therefore, in the present study, the antibacterial activity of the as-prepared noncovalently functionalized graphene aqueous dispersion against a Gram-positive (Staphylococcus aureus) and a Gram-negative (Escherichia coli) strain is evaluated and compared with that of the GO analogue.

\section{Results and discussion}

\section{Graphene (G) dispersion inside liposomal bilayers in aqueous solution}

Homogeneous aqueous suspensions of graphene-loaded liposomes (LIPO-G) were prepared by sonication of graphite in the presence of liposomes. The exfoliation was monitored by UV-Vis absorption spectroscopy in the $200-800 \mathrm{~nm}$ wavelength range. The observed flat and featureless spectrum is typical for the exfoliated graphitic material, ${ }^{50}$ absorption from liposomes being negligible above $400 \mathrm{~nm}$ (see Fig. 1).

Such an observation allowed the use of suspension absorbance at $660 \mathrm{~nm}$ as a measure of the concentration of exfoliated graphene loaded in the liposomal bilayer. The quantity of graphene was estimated from the absorbance at $660 \mathrm{~nm}$ by using the extinction coefficient $\left(\varepsilon=1390 \mathrm{mg}^{-1} \mathrm{~mL} \mathrm{~m}^{-1}\right)$ previously determined in water. ${ }^{50}$ With the designed protocol of graphene exfoliation and dispersion inside the liposomal bilayer, a yield of $15.6 \pm 1.0 \%$ in PBS $\left([\mathrm{G}]=0.161 \pm 0.010 \mathrm{mg} \mathrm{mL}^{-1}\right)$ and $13.2 \pm 0.4 \%$ in Milli-Q water $\left([\mathrm{G}]=0.124 \pm 0.010 \mathrm{mg} \mathrm{mL}^{-1}\right)$ was obtained. Whereas a

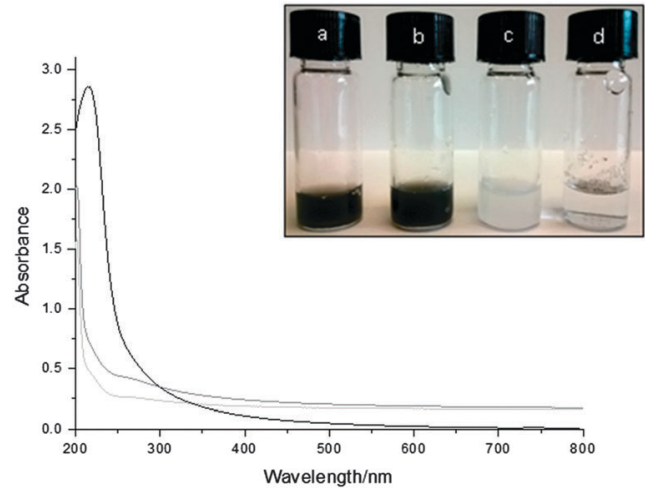

Fig. 1 UV-Vis spectra of graphene-loaded liposomes (LIPO-G) in PBS (light grey line), in Milli-Q water (dark grey line) and empty liposomes (black line) used as baseline. Inset: Digital pictures of vials containing LIPO-G in Milli-Q water (a) and in PBS (b), empty liposomes in PBS (c) and graphite in Milli-Q water after sonication for $2 \mathrm{~h}$ in the absence of phospholipids (d). graphene entrapment efficiency of $1.9 \pm 0.1 \%$ of the investigated liposomes (i.e. [weight of G]/[weight of phospholipids]\%) could be calculated in PBS.

Dynamic light scattering experiments revealed that the as-prepared LIPO-G had diameters in the $240-310 \mathrm{~nm}$ range (i.e. $238 \pm 6 \mathrm{~nm}$ for samples in PBS and $307 \pm 3 \mathrm{~nm}$ for samples in Milli-Q water), which is the typical feature of large unilamellar vesicles (LUV). A diameter of $222 \pm 7 \mathrm{~nm}$ was measured for POPC liposomes without graphene.

The $\zeta$-potential measurements showed that LIPO-G had an average negative $\zeta$-potential of $-15.2 \pm 0.3 \mathrm{mV}$. Pure POPC liposomes without graphene had a comparable $\zeta$-potential $(-15.4 \pm 0.8 \mathrm{mV})$ whereas the $\zeta$-potential of pristine graphene in water is $-21.8 \pm 0.05 \mathrm{mV} .^{51,52}$ All the above evidence pointed to the perfectly sandwiched graphene in the liposomal bilayer.

\section{Stability of graphene-loaded liposomes}

Liposome dimensions (and the relevant polydispersity indices) ${ }^{53}$ were checked over $48 \mathrm{~h}$ (see Fig. S1 in the ESI $\dagger$ ) and the corresponding dispersions were demonstrated to be relatively stable over time. It is worth noting that polydispersity of the obtained LIPO-G ranged between 0.25 and 0.30 , underlying a fairly homogeneous liposomal population once considered that sonication was the only homogenization step performed. ${ }^{53}$

The $\zeta$-potential is often considered a further measure of the colloidal stability of a suspension, in particular, $\zeta$-potential higher than $+15 \mathrm{mV}$ or lower than $-15 \mathrm{mV}$ are indicative of stable colloidal samples. ${ }^{54}$ The $\zeta$-potential for the obtained samples ranged between -15.7 and $-14.8 \mathrm{mV}$ during $48 \mathrm{~h}$ (see Fig. S2 in the ESI $\dagger$ ).

In view of potential administration in a biological environment another parameter to consider for materials intended to be used in biological samples is the stability upon dilution. As reported in Fig. S3 in the ESI, $\dagger$ the stability of the diluted samples overwhelmed that of the as-prepared samples either in terms of dimensions or polydispersity index.

\section{Evidence of graphite exfoliation}

Raman spectroscopy was used to confirm the exfoliation of graphite in few-layer graphene by exploiting POPC phospholipids. The Raman spectra of graphene showed the $G$ peak located at $\sim 1580 \mathrm{~cm}^{-1}$ and the 2D peak at $\sim 2700 \mathrm{~cm}^{-1}$ (see Fig. 2). The small D peak located at $\sim 1350 \mathrm{~cm}^{-1}$ is due to the firstorder zone boundary phonons and is indicative of the presence of some defects in the exfoliated graphene (see Fig. 2). It is accepted that Raman spectroscopy could be used to quantify the degree or type of defects of the exfoliated graphene and determine the number of layers by monitoring the shape, width, and position of the $2 \mathrm{D}$ peak. ${ }^{55}$

The Lorentzian fitting of the 2D Raman band allowed us to ascertain that treatment with liposomal suspension caused good exfoliation of graphite into a few-layer graphene. ${ }^{56}$ As a matter of fact, fitting of $2 \mathrm{D}$ peaks recorded over 14 different spots highlighted the presence of double-layer graphene (see Fig. 3 as an example and Fig. S4 and S5 in the ESI $\dagger$ ). 


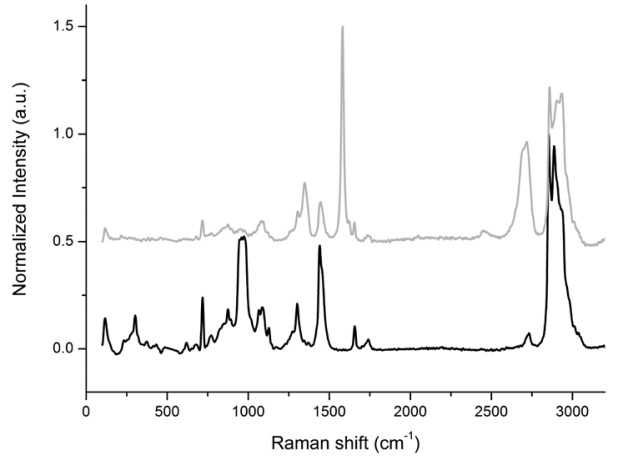

Fig. 2 Raman spectra of liposomal suspension (black line) and LIPO-G (grey line). The peak at $1000 \mathrm{~cm}^{-1}$ is due to the silicon wafer

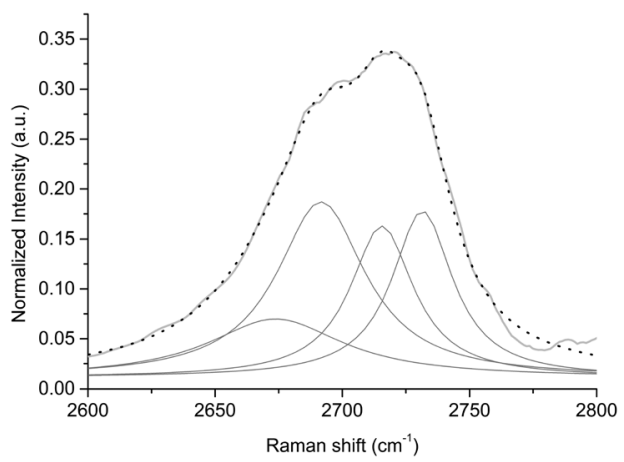

Fig. 3 Example of the fitting of the double layer graphene line shape with four Lorentzian components. Solid light grey line: experimental Raman spectrum; solid dark grey lines: four Lorentzian components and dotted black line: fitting of the four components.

Raman spectroscopy is also particularly useful for investigating fluidity and conformational order in a liposomal bilayer ${ }^{57-60}$ as, in POPC liposomes, the assignment of the peaks is wellestablished. ${ }^{61-63}$ According to the literature ${ }^{63,64}$ Raman intensity ratios $I_{2895} / I_{2855}, I_{2931} / I_{2895}$ and $I_{1125} / I_{1086}$ can be considered as good indicators of the molecular order of the lipid bilayer. Indeed, the $I_{1125} / I_{1086}$ Raman intensity ratio is related to the average number of "trans" bonds in the acyl chain, thus giving a measure of the order in the intra-chain structure. In contrast, the $I_{2895} / I_{2855}$ Raman intensity ratio, depending on the vibrational coupling between the adjacent POPC alkyl chains, reveals the lateral interactions between lipid chains whereas $I_{2931} / I_{2895}$ reflects both the inter- and intra-chain order/disorder features.

The inclusion of graphene into the bilayer showed a slight increase of the order of the liposomal system as demonstrated by the increase of both $I_{2931} / I_{2895}$ (from 0.87 to 0.89 ) and $I_{2895} / I_{2855}$ (from 0.84 to 1.05 ) values. The latter intensity ratio increase was unexpected because interactions with guests penetrating the bilayer is generally associated with a decrease of $I_{2895} / I_{2855}{ }^{65}$ Likely, the presence of graphene did not hamper inter-chain interactions and therefore bilayer self-assembly (i.e. the $I_{2931} / I_{2895}$ ratio did not vary), but favoured additional interactions between alkyl chains of POPC molecules and graphene layers. ${ }^{66,67}$ Such evidence pointed to the prevailing arrangement of exfoliated graphene inside the core of the bilayer in perfect agreement with coarse-grained
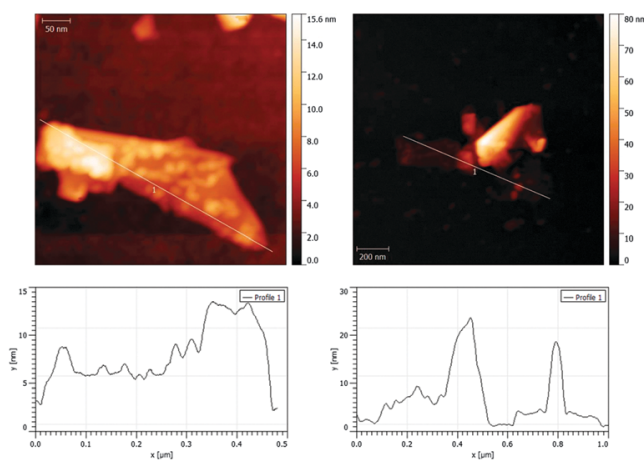

Fig. 4 AFM images of LIPO-G (upper images) and cross-section along the cross line (bottom images). Scale bars are: $50 \mathrm{~nm}$ (upper left) and $200 \mathrm{~nm}$ (upper right).

dynamic simulations that showed graphene perfectly sandwiched into POPC bilayers and almost not affecting its thickness. ${ }^{45}$ Graphene bilayers slowly diffused in the membrane interior, but the composite system stayed stable over time. Accordingly, the Raman intensity ratio $I_{1125} / I_{1086}$ decreased thus denoting a decrease in the average number of "trans" bonds in the alkyl chains of POPC as a consequence of folding of the alkyl chains to promote van der Waals interactions between alkyl chains and the graphene surface.

In order to further confirm the exfoliation degree of graphene, we performed AFM analysis. Fig. 4 reports AFM images of LIPO-G and the corresponding cross-sectional view of samples along the cross line. The samples, prepared by drop-casting a dilute LIPO-G solution onto a silicon wafer, were then dried under vacuum and washed with methanol to get rid of excess liposomes and phospholipids. The cross-sectional AFM view showed that the minimum height of the liposome coated sample is ca. 3-4 nm. Since: (i) the sample is formed of graphene covered with variable amounts of phospholipids and is therefore a difficult sample to be analyzed by AFM due to the dependence of the AFM measurements on the roughness and cleanness of the surface, ${ }^{68}$ (ii) the thickness of a phospholipid bilayer is around $0.4 \mathrm{~nm},{ }^{69}$ (iii) stabilizers cannot touch the surface of graphene directly, ${ }^{68,70}$ and a gap exists between phospholipids and graphene, and (iv) AFM measurements depend strongly on the substrate used for graphene deposition and are distorted by the variability of substrates, ${ }^{68,71,72}$ these data supported the formation of fewlayer graphene, likely with not more than 2-3 layers.

Fig. 5 presents typical TEM images of the analyzed sample. The results showed that vesicles belong to the class of LUVs with a mean diameter of about 150-180 $\mathrm{nm}$ for empty liposomes (Fig. 5A) and 240-280 nm for LIPO-G (Fig. 5B-D). While LIPO-G LUVs were easily observed without the use of vesicle staining, empty LUVs were difficult to focalize and visualize on the TEM grid. The loading of graphene did not significantly alter LUV morphology and, during the experiments, LIPO-G LUVs appeared to be able to stand the electron beam for longer time with respect to empty liposomes. Fig. 5B-D clearly evidence the presence of graphene sheets inside the liposomes although, due to the need to eliminate the solvent, the real disposition of graphene 

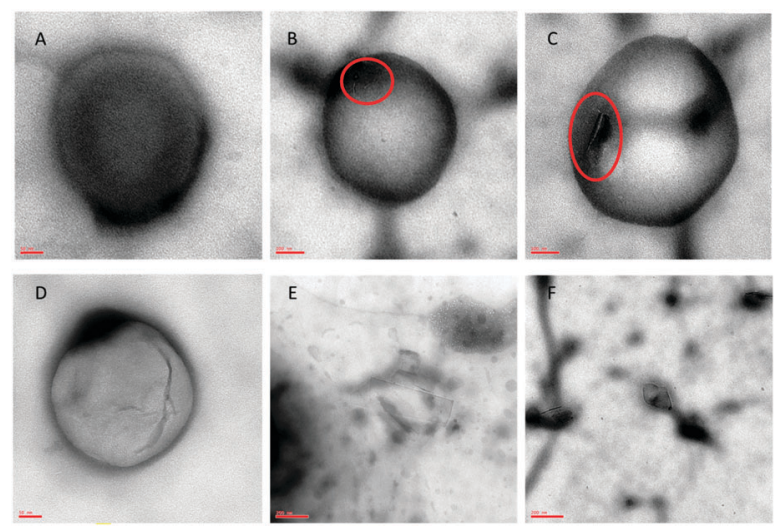

Fig. 5 TEM images of empty liposomes magnified at $140000 \times(A)$, LIPO$G$ magnified at $85000 \times(B$ and $C$ ) and $140000 \times(D)$ and graphene sheets magnified at $50000 \times$ ( $E$ and F). Scale bars are: $200 \mathrm{~nm}$ for $50000 \times$ images, $100 \mathrm{~nm}$ for $85000 \times$ images and $50 \mathrm{~nm}$ for $140000 \times$ images. All samples were performed in Milli- $Q$ water. Red circles indicate partially hindered graphene sheets.

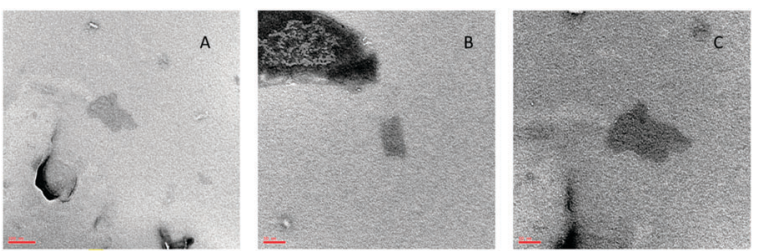

Fig. 6 TEM images of graphene flakes in solution obtained by degradation of liposomes with Triton X-100. Some impurities due to the presence of Triton X-100 and isolated phospholipids are clearly detectable in the samples. The adsorption of phospholipids and surfactants could partly explain the colour contrast of the flakes. (A) Magnification of $85000 \times$ and $140000 \times(B$ and C). Scale bars are: $100 \mathrm{~nm}$ for $85000 \times$ images and $50 \mathrm{~nm}$ for $140000 \times$ images. All samples were performed in Milli-Q water.

with respect to the not dried bilayer was difficult to envisage. Only very few graphene flakes were observed outside the liposomes deposited onto the TEM grid (Fig. 5E and F). In order to ascertain that graphene was inserted inside the liposomes we destroyed the liposomes by addition of $10 \mu \mathrm{L}$ of a concentrated Triton X-100 aqueous solution to the liposomal solution. The precipitation of graphene flakes was detectable by the eye (see Fig. S6 in the ESI $\dagger$ ). The so-obtained solution was deposited onto a copper grid and visualized by TEM. Several very small $(c a .100 \mathrm{~nm})$ graphene flakes could be detected this time on the grid (Fig. 6).

The size and size distribution of LUVs in the as-prepared dispersion were also checked by DLS experiments and the results were found to be in good agreement with TEM measurements (see previously reported data).

\section{Antimicrobial activity}

Staphylococcus aureus and Escherichia coli were used to evaluate the antibacterial effect of graphene oxide (GO) and LIPO-G at the

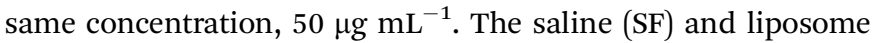
suspension (LIPO) without graphene were used as controls. The antibacterial activity of LIPO-G, compared to that recently reported $^{73}$ for GO with respect to the control LIPO and SF, respectively, after $2 \mathrm{~h}$ of contact, is shown in Fig. 7 .

The number of colony forming units of $S$. aureus ATCC 29213 and $E$. coli ATCC 8739 was slightly reduced in the presence of both GO and LIPO-G when compared to the CFUs detected with saline and LIPO. This reduction was significant $(P<0.05)$ in $S$. aureus after GO and LIPO-G treatment $v s$. SF, as well as in E. coli after GO and LIPO-G treatment vs. SF (Fig. 7A). The reduction of CFUs is clearly observed on TSA and LB agar plates (Fig. 7B) where the colony count was performed.

Fig. 8 displays the reduction of bacterial growth with the drop methodology. After $24 \mathrm{~h}$ of incubation in the presence of GO and LIPO-G, both $S$. aureus and E. coli colonies were reduced with respect to the controls.

The microscopic analysis of bacterial colonies confirmed the goodness of the reading. In fact, the observed bacteria do not aggregate with each other underlying that each counted colony is derived from one singular bacterial cell (see Fig. S7 in the $\mathrm{ESI} \dagger$ ).

The percentage of reduction of bacterial growth in the presence of GO and LIPO-G, obtained by colony count (see Fig. 7), is reported in Table 1.

Values of bacterial growth reduction of $91.0 \%$ for $S$. aureus and $91.9 \%$ for $E$. coli were recorded after treatment with GO with respect to the control (SF) in agreement with literature data. ${ }^{73}$ Instead reduction of $60.1 \%$ for $S$. aureus and $78.5 \%$ for E. coli were recorded after treatment with LIPO-G with respect to control (LIPO). It is worth noting that treatment with LIPO-G, with respect to $\mathrm{SF}$, induced a reduction of $88.4 \%$ and $86.5 \%$, respectively, for $S$. aureus and $E$. coli, highlighting an antimicrobial activity comparable to that reported for hydrophilic GO. These data are particularly interesting because they point out the ability of liposomes to mediate the antimicrobial activity of the hydrophobic and non-covalently functionalized graphene.

No significant selectivity of LIPO-G is detected for Grampositive or Gram-negative bacteria although an almost complete inhibition of growth of Gram-positive bacteria is monitored with respect to $t=0$. This information is particularly interesting as GO evidenced instead a killing effect.

As far as the mechanism of antibacterial activity is concerned, it is likely, following previously published papers, ${ }^{42,43}$ and the observed affinity of pure liposomes and LIPO-G for the external membrane of the cell wall of Gram-negative organisms, that damage to the membrane was involved. Indeed, pure liposomes demonstrated to stimulate mostly the growth of Gram-negative bacteria with respect to Gram-positive bacteria due to the similar phospholipid composition of the external membrane of their cell wall. Analogously, graphene non-covalently functionalized with phospholipids reduced the growth of Gram-negative bacteria twice as much as that of Gram-positive bacteria.

The bacterial viability is reported, by using live/dead staining, in Fig. 9. Both Staphylococcus aureus and Escherichia coli cells were uniformly viable as indicated by a green fluorescence with only a few red dead cells.

Taken together these data confirmed that the main effect obtained after contact with GO and LIPO-G was bacterial growth 
A
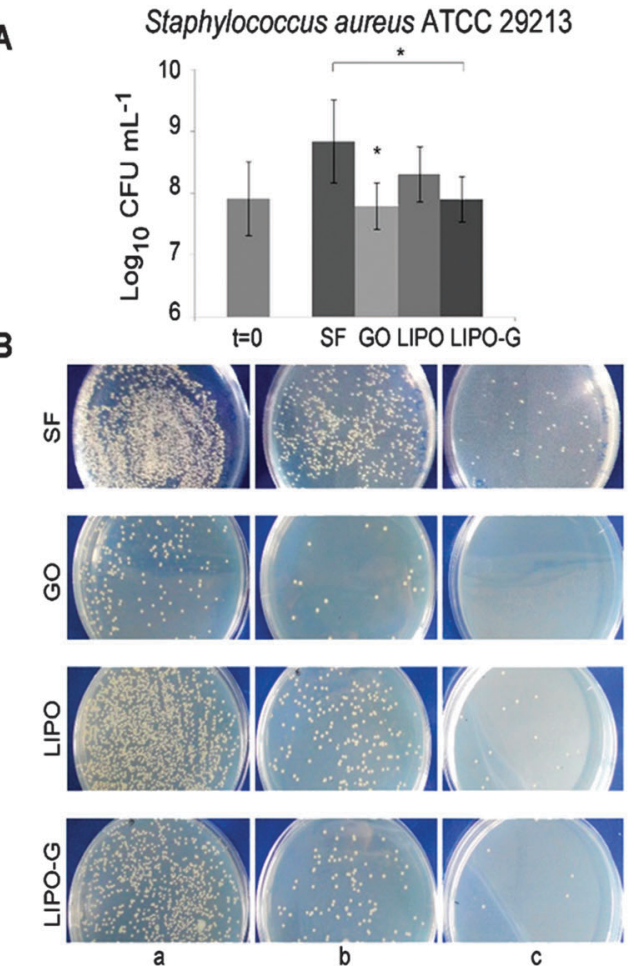

Escherichia coli ATCC 8739
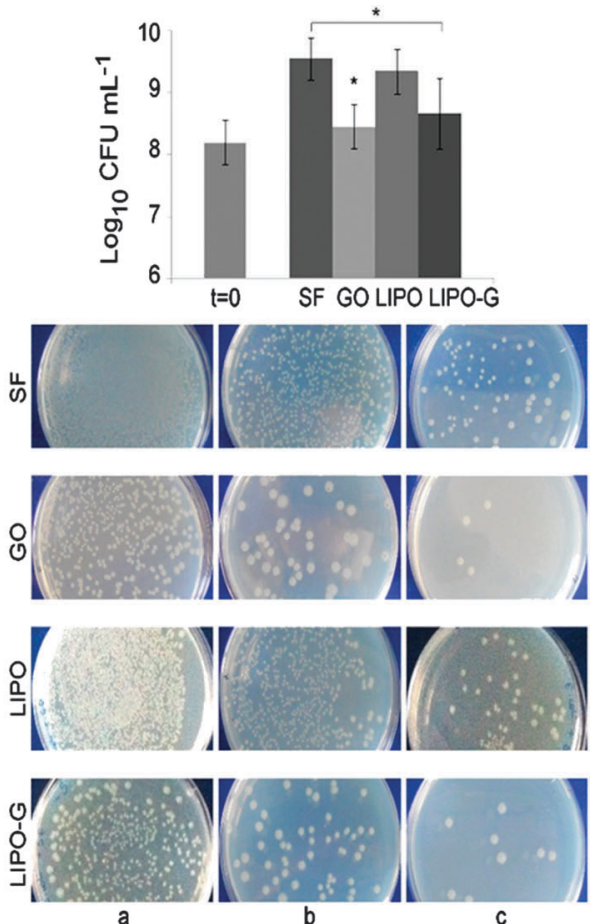

Fig. 7 Effect of graphene oxide (GO) and graphene-embedded in liposomal suspension (LIPO-G) on the growth of Staphylococcus aureus ATCC 29213 and Escherichia coli ATCC 8739 compared to the respective controls. (A) Bacterial viable count; (B) representative TSA and LB agar plates for the bacterial viable count (a-c - serial dilutions). * Significant reduction $(P<0.05)$.

Staphylococcus aureus ATCC 29213
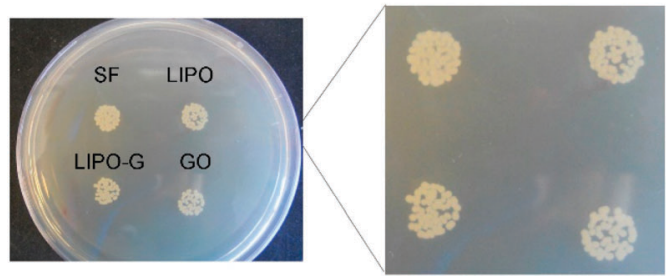

Escherichia coli ATCC 8739
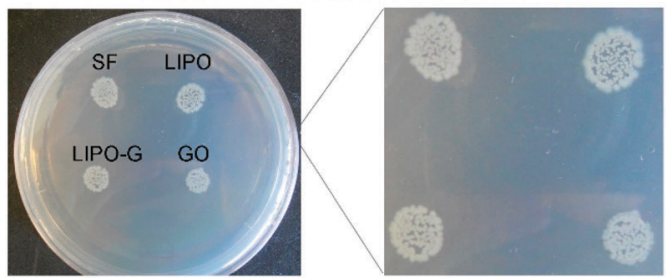

Fig. 8 Effect of GO and LIPO-G on S. aureus ATCC 29213 and E. coli ATCC 8739 growth after $24 \mathrm{~h}$ of contact. The enlarged images clearly show the lower number of colonies detected compared to the controls.

inhibition and a very low killing effect. In fact, after $2 \mathrm{~h}$ of incubation, the bacteriostatic effect was clearly detectable by the smaller number of bacteria per field with respect to the bacterial population in the controls. Moreover, these treated bacteria appeared almost totally viable with only a few dead red cells strongly suggesting the bacteriostatic effect of GO and LIPO-G on both S. aureus and E. coli.

\section{Experimental}

\section{Materials}

Graphite powder $(99.99 \%, 200$ mesh) was purchased from Alfa Aesar and 1-palmitoyl-2-oleyl-sn-glycero-3-phosphocholine (POPC) was purchased from Avanti Polar Lipids, Inc. (Alabaster, AL). Graphene oxide (GO) used for comparative antibacterial activity determination was prepared by using the modified Hummers method. ${ }^{40,74}$

Unless otherwise stated, reagents were of analytical grade and used as received. All aqueous solutions were prepared using Ultrapure Milli-Q water (electric resistance $>18.2 \mathrm{M} \Omega \mathrm{cm}^{-1}$ ) from a Millipore Corp. model Direct-Q 3 system.

\section{Apparatus}

Sonication was performed by using an ultrasonic bath (Transsonic 310 Elma, $35 \mathrm{kHz}$ ). UV-Vis spectra were recorded on a Jasco V-550. Measurement of vesicle size and $\zeta$-potential values was performed by using a 90Plus/BI-MAS Zeta Plus multiangle particle size analyzer (Brookhaven Instruments Corp.). Raman spectra were recorded on an Invia Renishaw microspectrometer $(50$ or $100 \times$ ) and a laser source at $532 \mathrm{~nm}$ (power $5 \%, 3$ or 5 accumulations/measurement). TEM observations were carried out on a Zeiss electron microscope M109 operated at $80 \mathrm{~kW}$ working voltage. The surfaces were imaged at a scan rate from 0.1 to $1.95 \mathrm{~Hz}$. Images were acquired using a Gatan camera (U.S.) and processed by Nanoscope Analysis software. AFM analysis was performed on a Nanoscope V (Digital Instruments Metrology 
Table 1 Percentage of growth reduction of Staphylococcus aureus ATCC 29213 (SA) and Escherichia coli ATCC 8739 (EC) after treatment with GO and LIPO-G compared with the respective controls (SF and LIPO)

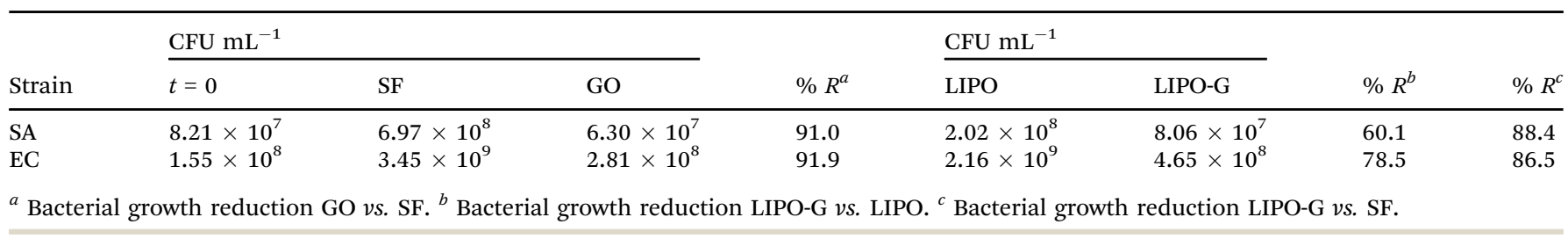
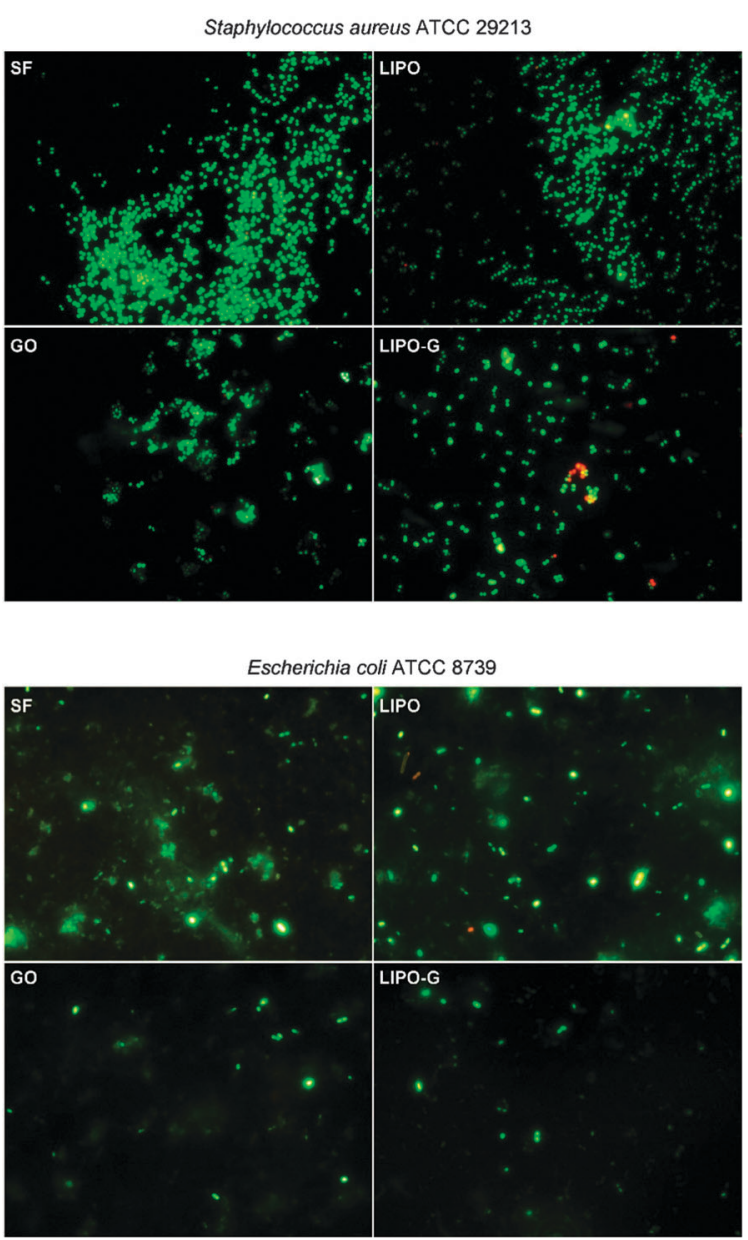

Fig. 9 Representative fluorescence images (Live/Dead staining) showing the microbial viability after $2 \mathrm{~h}$ treatment with SF, GO, LIPO and LIPO-G. Green bacterial cells are viable and red bacterial cells are dead. Images obtained from a fluorescence optical microscope (Leica Microsystems Spa, Milan, Italy) functionalized at $1000 \times$.

Group, model MMAFMLN) in tapping mode in air at room temperature, using an n-type silicon $\mu$ mash $^{\circledR}$ SPM probe (HQ:NSC15/AL BS) with 12-18 $\mu \mathrm{m}$ tip height and $<40^{\circ}$ cone angle (resonant frequency of $325 \mathrm{kHz}$ and force constant of $\sim 40 \mathrm{~N} \mathrm{~m}^{-1}$ ). The collected images were then analyzed using WsXm 4.0 software (Nanotec Electronica S. L.) and Gwyddion 2.39.

\section{Preparation and processing of graphene-loaded liposomes}

Liposomes were prepared according to the following protocol. $40 \mathrm{mg}$ of POPC, dissolved in $4 \mathrm{~mL}$ of chloroform, were put in a round-bottomed flask and dried in a rotary evaporator under reduced pressure at $40{ }^{\circ} \mathrm{C}$ to form a thin lipid film on the inside wall of the flask. The phospholipid film was kept at $4{ }^{\circ} \mathrm{C}$ overnight before rehydration with $5 \mathrm{~mL}$ of Milli-Q water or PBS buffer ( $\mathrm{pH} 7.4,304 \mathrm{mOsm}$ ) in order to obtain a $0.8 \% \mathrm{w} / \mathrm{w}$ aqueous solution of phospholipid, a concentration that is above the critical aggregation concentration value of POPC $(0.1 \% \mathrm{w} / \mathrm{w}) .5 \mathrm{mg}$ of graphite powder was added to the resulting liposome suspension and sonicated for $2 \mathrm{~h}$. The resulting mixture was centrifuged at $5000 \mathrm{rpm}$ for $15 \mathrm{~min}$ to sediment unexfoliated particles or thick flakes of graphite and the supernatant, which was the final LIPO-G suspension, was collected.

The exfoliation yield and the encapsulation efficiency of LIPO-G were calculated by UV-Vis spectrophotometry by determining the amount of graphene present in the supernatant after liposomal centrifugation.

The liposomal suspension was characterized by using UV-Vis spectroscopy, dynamic light scattering (DLS) experiments, $\zeta$-potential measurements, transmission electron microscopy (TEM), Raman spectroscopy and AFM. The samples for Raman characterization were obtained by dropping the liposomal suspension on a wafer surface (we used n-type doped prime $\mathrm{SiO}_{2}$ wafers) and leaving the sample in the oven at $40{ }^{\circ} \mathrm{C}$ for $24 \mathrm{~h}$ in order to dry the aqueous solution. TEM samples were prepared by dropping a small drop of liposomal suspension (about $5 \mu \mathrm{L}$ ) on a carbon coated copper grid. Solvent was removed by keeping the grid at $30^{\circ} \mathrm{C}$ for $60 \mathrm{~h}$ in an incubator in the presence of silica gel in order to ensure a total absence of moisture. The choice of using a low drying temperature for a relatively long time is correlated with the need to avoid high temperature and thus graphene sheet folding. AFM samples were prepared by drop-casting the solution onto silicon wafer substrates, drying under vacuum and washing the sample with methanol to remove excess liposomes and phospholipids.

\section{Bacterial culture}

Staphylococcus aureus ATCC 29213 and Escherichia coli ATCC 8739 were used in the present study. Staphylococcus aureus and E. coli were cultured in Trypticase Soy broth (TSB, Liofilchem, Italy) and Luria Bertani broth (LB, Oxoid, Italy) media, respectively, and incubated at $37{ }^{\circ} \mathrm{C}$ overnight under aerobic conditions. Cultures were diluted $1: 10(\mathrm{v} / \mathrm{v})$ in the respective TSB and LB media and refreshed for $2 \mathrm{~h}$ at $37{ }^{\circ} \mathrm{C}$ in an orbital shaker under aerobic conditions to obtain an exponential growth phase with a homogeneous bacterial population. Subsequently, the broth-cultures were washed in PBS ( $\mathrm{pH}$ 7.2) and adjusted to an optical density $\left(\mathrm{Abs}_{600}\right)$ of 0.12 corresponding to approximately $5 \times 10^{7} \mathrm{CFU} \mathrm{mL}^{-1}$ and used for the experiments. 


\section{Antimicrobial activity}

The standardized broth-cultures of S. aureus ATCC 29213 and E. coli ATCC 8739 were incubated with the same final concentration of $50 \mu \mathrm{g} \mathrm{mL} \mathrm{m}^{-1} \mathrm{GO}$, used as a comparison for the antibacterial activity of pristine graphene, and graphene-embedded in liposomal suspension (LIPO-G); as controls, bacteria were incubated with fresh isotonic saline solution (SF) and an equally concentrated POPC liposomal suspension (LIPO) at $37{ }^{\circ} \mathrm{C}$ at $250 \mathrm{rpm}$ in an orbital shaker for $2 \mathrm{~h}$. The loss of bacterial viability was evaluated by counting the colony forming units (CFUs) using a Colony Counter Star-Count STC 1000 (VWR International PBI Srl, Via San Giusto, Milan, Italy). Briefly, a series of 10 -fold cell dilutions $(100 \mu \mathrm{L})$ were spread onto Trypticase Soy Agar (TSA, Liofilchem, Italy) plates for $S$. aureus and onto LB agar (LB, Oxoid, Italy) plates for E. coli and incubated for $24 \mathrm{~h}$ at $37^{\circ} \mathrm{C}$. The cell growth inhibition was detected by comparing the colony counts between GO vs. SF and LIPO-G vs. LIPO.

All experiments were performed in duplicate, and repeated for at least three independent experiments. The significance of the differences recorded in the experiments performed with SF, GO, LIPO, LIPO-G was evaluated using Student's $t$-test. Probability levels $<0.05$ were considered statistically significant.

The inhibition of CFU formation was also performed by dropping $10 \mu \mathrm{L}$ of test substances (i.e. SF, GO, LIPO, LIPO-G) on agar plates with $10 \mu \mathrm{L}$ of standardized bacterial suspensions. Plates were incubated at $37{ }^{\circ} \mathrm{C}$ for $24 \mathrm{~h}$ (see Table 1).

After treatment with SF, GO, LIPO, LIPO-G for $2 \mathrm{~h}$, the bacterial viability was also evaluated using a Live/Dead Kit (Molecular Probes Inc., Invitrogen, Italy) as indicated by the manufacturer and visualized under a fluorescence Leica 4000 DM microscope (Leica Microsystems Spa, Milan, Italy). Ten fields of view randomly chosen for each slide were observed. Microscopic observations were repeated for three independent experiments.

\section{Conclusions}

We present a facile and prompt exfoliation protocol for graphite by exploiting $2 \mathrm{~h}$ of sonication of graphite in POPC large unilamellar vesicle aqueous solution. Spectrophotometry was used to quantify the exfoliated graphene. Raman spectroscopy demonstrated the prevailing formation of double-layer graphene and AFM confirmed the formation of few-layer graphene. TEM pointed out that sonication allows the formation of nanometric few-layer graphene sheets embedded in liposomes. The corresponding aqueous solution demonstrated to form a solution stable for hours and with significant antibacterial activity. In particular, data evidenced that liposome-embedded graphene reduces the growth capability of bacteria. In particular, it almost completely inhibits the microbial growth of Gram-positive bacteria whereas Gram-negative bacterial growth is reduced to a mere three-fold.

\section{Acknowledgements}

The authors thank Dr Patrizia De Marco and Dr Antonello Di Crescenzo of the University "G. d'Annunuzio" of Chieti-Pescara for useful discussion on Raman and TEM analyses, and Prof. Maurizio Prato of the University of Trieste for useful discussion. The authors wish to thank University 'G. d'Annunzio' of Chieti-Pescara, University of Trieste, MIUR (PRIN 2010-11, prot. 2010N3T9M4 and FIRB, prot. RBAP1095CR) and POR FESR Abruzzo 2007-2013 for financial support. R.Z. thanks Regione Abruzzo (Reti per l'alta formazione - P.O. F.S.E. Abruzzo 20072013) that funded her annual postdoctoral fellowship.

\section{Notes and references}

1 M. J. Allen, V. C. Tung and R. B. Kaner, Chem. Rev., 2010, 110, 132.

2 A. K. Geim, Science, 2009, 324, 1530.

3 A. C. Ferrari, F. Bonaccorso, V. Fal'ko, K. S. Novoselov, S. Roche, P. Bøggild, S. Borini, F. H. L. Koppens, V. Palermo, N. Pugno, J. A. Garrido, R. Sordan, A. Bianco, L. Ballerini, M. Prato, E. Lidorikis, J. Kivioja, C. Marinelli, T. Ryhänen, A. Morpurgo, J. N. Coleman, V. Nicolosi, L. Colombo, A. Fert, M. Garcia-Hernandez, A. Bachtold, G. F. Schneider, F. Guinea, C. Dekker, M. Barbone, Z. Sun, C. Galiotis, A. N. Grigorenko, G. Konstantatos, A. Kis, M. Katsnelson, L. Vandersypen, A. Loiseau, V. Morandi, D. Neumaier, E. Treossi, V. Pellegrini, M. Polini, A. Tredicucci, G. M. Williams, B. Hee Hong, J.-H. Ahn, J. Min Kim, H. Zirath, B. J. van Wees, H. van der Zant, L. Occhipinti, A. Di Matteo, I. A. Kinloch, T. Seyller, E. Quesnel, X. Feng, K. Teo, N. Rupesinghe, P. Hakonen, S. R. T. Neil, Q. Tannock, T. Löfwander and J. Kinaret, Nanoscale, 2015, 7, 4598.

4 T. Cohen-Karni, Q. Qing, Q. Li, Y. Fang and C. M. Lieber, Nano Lett., 2010, 10, 1098.

5 P. Begum, R. Ikhtiari and B. Fugetsu, Carbon, 2011, 49, 3907.

6 K. Bourzac, Nature, 2012, 483, S34.

7 C. Schmidt, Nature, 2012, 483, S37.

8 F. Bonaccorso, Z. Sun, T. Hasan and A. C. Ferrari, Nat. Photonics, 2010, 4, 611.

9 N. Savage, Nature, 2012, 483, S38.

10 S. Zhu, S. Tang, J. Zhang and B. Yang, Chem. Commun., 2012, 48, 4527.

11 X. Huang, X. Qi, F. Boey and H. Zhang, Chem. Soc. Rev., 2012, 41, 666.

12 J.-Y. Hong and J. Jang, J. Mater. Chem., 2012, 22, 8179.

13 L. Dai, Acc. Chem. Res., 2013, 46, 31.

14 Y. Song, W. Wei and X. Qu, Adv. Mater., 2011, 23, 4215.

15 Y. Liu, X. Dong and P. Chen, Chem. Soc. Rev., 2012, 41, 2283.

16 S. Sayyar, E. Murray, B. C. Thompson, J. Chung, D. L. Officer,

S. Gambhir, G. M. Spinks and G. G. Wallace, J. Mater. Chem. $B, 2015,3,481$.

17 H. Zhang, G. Grüner and Y. Zhao, J. Mater. Chem. B, 2013, 1, 2542.

18 C. Chung, Y.-K. Kim, D. Shin, S.-R. Ryoo, B. H. Hong and D.-H. Min, Acc. Chem. Res., 2013, 46, 2211.

19 X. M. Sun, Z. Liu, K. Welsher, J. T. Robinson, A. Goodwin, S. Zaric and H. J. Dai, Nano Res., 2008, 1, 203. 
20 Z. Liu, J. T. Robinson, X. M. Sun and H. J. Dai, J. Am. Chem. Soc., 2008, 130, 10876.

21 K. Yang, S. A. Zhang, G. X. Zhang, X. M. Sun, S. T. Lee and Z. A. Liu, Nano Lett., 2010, 10, 3318.

22 Y. Wang, Z. H. Li, D. H. Hu, C. T. Lin, J. H. Li and Y. H. Lin, J. Am. Chem. Soc., 2010, 132, 9274.

23 S. J. He, B. Song, D. Li, C. F. Zhu, W. P. Qi, Y. Q. Wen, L. H. Wang, S. P. Song, H. P. Fang and C. H. Fan, Adv. Funct. Mater., 2010, 20, 453.

24 C. H. Lu, H. H. Yang, C. L. Zhu, X. Chen and G. N. Chen, Angew. Chem., Int. Ed., 2009, 48, 4785.

25 X. H. Zhao, R. M. Kong, X. B. Zhang, H. M. Meng, W. N. Liu, W. H. Tan, G. L. Shen and R. Q. Yu, Anal. Chem., 2011, 83, 5062.

26 H. X. Chang, L. H. Tang, Y. Wang, J. H. Jiang and J. H. Li, Anal. Chem., 2010, 82, 2341-2346.

27 C. Peng, W. B. Hu, Y. T. Zhou, C. H. Fan and Q. Huang, Small, 2010, 6, 1686.

28 R. Kempaiah, A. Chung and V. Maheshwari, ACS Nano, 2011, 5, 6025.

29 P. Nguyen and V. Berry, J. Phys. Chem. Lett., 2012, 3, 1024.

30 W. C. Lee, C. H. Lim, H. Shi, L. A. Tang, Y. Wang, C. T. Lim and K. P. Loh, ACS Nano, 2011, 5, 7334.

31 Y. Luo, H. Shen, Y. Fang, Y. Cao, J. Huang, M. Zhang, J. Dai, X. Shi and Z. Zhang, ACS Appl. Mater. Interfaces, 2015, 7, 6331.

32 T.-H. Kim, S. Shah, L. Yang, P. T. Yin, Md. K. Hossain, B. Conley, J.-W. Choi and K. B. Lee, ACS Nano, 2015, 9, 3780.

33 B. Chaudhuri, D. Bhadra, L. Moroni and K. Pramanik, Biofabrication, 2015, 7, 1.

34 S. H. Ku and C. B. Park, Biomaterials, 2013, 34, 2017.

35 C. Zhao, X. Lu, C. Zanden and J. Liu, Biomed. Mater., 2015, 10, 015019.

36 R. Subbiah, P. Du, S. Y. Van, M. Suhaeri, M. P. Hwang, K. Lee and K. Park, Biomed. Mater., 2014, 9, 065003.

37 S. Y. Park, J. Park, S. H. Sim, M. G. Sung, K. S. Kim, B. H. Hong and S. Hong, Adv. Mater., 2011, 23, H263.

38 G. Y. Chen, D. W. Pang, S. M. Hwang, H. Y. Tuan and Y. C. Hu, Biomaterials, 2012, 33, 418.

39 B. C. Thompson, E. Murray and G. G. Wallace, Adv. Mater., 2015, DOI: 10.1002/adma.201500411.

40 V. Ettorre, P. De Marco, S. Zara, V. Perrotti, A. Scarano, A. Di Crescenzo, M. Petrini, C. Hadad, D. Bosco, B. Zavan, L. Valbonetti, G. Spoto, G. Iezzi, A. Piattelli, A. Cataldi and A. Fontana, ACS Appl. Mater. interfaces, submitted.

41 X. Zhou and F. Liang, Curr. Med. Chem., 2014, 21, 855.

42 O. Akhavan and E. Ghaderi, ACS Nano, 2010, 4, 5731.

43 W. B. Hu, C. Peng, W. J. Luo, M. Lv, X. Li, D. Li, Q. Huang and C. Fan, ACS Nano, 2010, 4, 4317.

44 S. Haar, A. Ciesielski, J. Clough, H. Yang, R. Mazzaro, F. Richard, S. Conti, N. Merstorf, M. Cecchini, V. Morandi, C. Casiraghi and P. Samori, Small, 2015, 11, 1691.

45 A. V. Titov, P. Kral and R. Pearson, ACS Nano, 2009, 4, 229. 46 W. Du, X. Jiang and L. Zhu, J. Mater. Chem. A, 2013, 1, 10592.
47 D. P. Speert, Can. J. Infect. Dis., 1996, 7, 169.

48 A. J. Huh and Y. J. Kwon, J. Controlled Release, 2011, 10, 128.

49 W. Gao, S. Thampiwatana, P. Angsantikul and L. Zhang, Wiley Interdiscip. Rev.: Nanomed. Nanobiotechnol., 2014, 6, 532.

50 U. Khan, A. O'Neil, M. Lotya, S. De and J. N. Coleman, Small, $2010,6,864$.

51 E. Sawosz, S. Jaworski, M. Kutwin, A. Hotowy, M. Wierzbicki, M. Grodzik, N. Kurantowicz, B. Strojny, L. Lipińska and A. Chwalibog, Int. J. Nanomed., 2014, 9, 3913.

52 Z. Jin, T. P. McNicholas, C.-J. Shih, Q. H. Wang, G. L. C. Paulus, A. J. Hilmer, S. Shimizu and M. S. Strano, Chem. Mater., 2011, 23, 3362.

53 J. Pereira-Lachataignerais, R. Pons, P. Panizza, L. Courbin, J. rouch and O. López, Chem. Phys. Lipids, 2006, 140, 88.

54 P. C. Hiemez and R. Rajagopalan, Principles of Colloid, Surface Chemistry, Marcel Dekker, New York, USA, 1997.

55 A. C. Ferrari, J. C. Meyer, V. Scardaci, C. Casiraghi, M. Lazzeri, F. Mauri, S. Piscanec, D. Jiang, K. S. Novoselov, S. Roth and A. K. Geim, Phys. Rev. Lett., 2006, 97, 187401.

56 I. Calizo, D. Teweldebrhan, W. Bao, F. Miao, C. N. Lau and A. A. Balandin, J. Phys.: Conf. Ser., 2008, 109, 012008.

57 J. S. Vincent, S. D. Revak, C. D. Cochrane and I. W. Levin, Biochemistry, 1993, 32, 8228.

58 B. J. Litman, E. N. Lewis and I. W. Levin, Biochemistry, 1991, 30, 313.

59 H. Takeuchi, Y. Nemoto and I. Harada, Biochemistry, 1990, 29, 1572.

60 F. Lhert, D. Blaudez, C. Heywang and J.-M. Turlet, Langmuir, 2002, 18, 512.

61 R. G. Snyder, H. L. Strauss and C. A. Elliger, J. Phys. Chem., 1982, 86, 5145.

62 D. Aslanian, M. Negrerie and R. Chambert, Eur. J. Biochem., 1986, 160, 395.

63 B. P. Gaber and W. L. Peticolas, Biochim. Biophys. Acta, Biomembr., 1977, 465, 260.

64 M. Picquart and T. Lefèvre, J. Raman Spectrosc., 2003, 34, 4. 65 M. Di Foggia, S. Bonora and V. Tugnoli, J. Therm. Anal. Calorim., 2013, 111, 1871.

66 Z. Derzkot and K. Jacobson, Biochemistry, 1980, 19, 6050. 67 J. S. Vincent and I. W. Levin, Biophys. J., 1991, 59, 1007.

68 F. Giannazzo, S. Sonde, V. Raineri, G. Patanè, G. Compagnini, F. Aliotta, R. Ponterio and E. Rimini, Phys. Status Solidi C, 2010, 7, 1251.

69 R. Zappacosta, A. Fontana, A. Credi, A. Arduini and A. Secchi, Asian J. Org. Chem., 2015, 4, 262.

70 C. Chen, W. Zhai, D. Lu, H. Zhang and W. Zheng, Mater. Res. Bull., 2011, 46, 583.

71 H. W. C. Postma, A. Sellmeijer and C. Dekker, Adv. Mater., 2000, 12, 1299.

72 Z. Su, Q. Fu, H. Li and K. Li, Nano, 2014, 12, 1450066.

73 S. Liu, T. H. Zeng, M. Hofmann, E. Burcombe, J. Wei, R. Jiang, J. Kong and Y. Chen, ACS Nano, 2013, 5, 6971.

74 T. Rattana and S. Chaiyakun, Procedia Eng., 2012, 32, 759. 